Kawada, Junzo (sous la direction de). - Cultures

sonores d'Afrique. Tokyo, University of Foreign Studies, Institut de recherches sur les langues et cultures d'Asie et d'Afrique, 1997, 393 p., bibl.

\title{
Silvia Paggi
}

\section{OpenEdition}

\section{Journals}

Édition électronique

URL : http://journals.openedition.org/etudesafricaines/1474

DOI : 10.4000/etudesafricaines. 1474

ISSN : 1777-5353

Éditeur

Éditions de l'EHESS

Édition imprimée

Date de publication : 1 janvier 2002

ISBN : 978-2-7132-1420-2

ISSN : 0008-0055

Référence électronique

Silvia Paggi, «Kawada, Junzo (sous la direction de). - Cultures sonores d'Afrique. Tokyo, University of Foreign Studies, Institut de recherches sur les langues et cultures d'Asie et d'Afrique, 1997, 393 p. bibl. », Cahiers d'études africaines [En ligne], 165 | 2002, mis en ligne le 25 mai 2005, consulté le 03 février 2021. URL : http://journals.openedition.org/etudesafricaines/1474 ; DOI : https://doi.org/ 10.4000/etudesafricaines. 1474

Ce document a été généré automatiquement le 3 février 2021

(c) Cahiers d'Études africaines 
Kawada, Junzo (sous la direction de). - Cultures sonores d'Afrique. Tokyo, University of Foreign Studies, Institut de recherches sur les langues et cultures d'Asie et d'Afrique, 1997, 393 p., bibl.

\section{Silvia Paggi}

1 Cet ouvrage réunit huit essais d'ethnomusicologie africaniste issus d'un projet de recherche développé entre 1994 et 1996, sous la direction du Professeur Junzo Kawada de l'Institut de recherches sur les langues et cultures d'Asie et d'Afrique de Tokyo. Un point de vue commun sous-tend ces textes : considérer que le terme «musique » est trop limité pour exprimer le vaste champ de la communication sonore en Afrique.

2 Les différents auteurs préfèrent donc employer le terme de "culture sonore " pour désigner un champ d'étude dont la musique, au sens occidental du terme, ne constitue que l'un des domaines. En outre, de toutes sortes de communication exprimées par le son, la «culture sonore» prend en compte également les aspects sociopolitiques du contexte dans lequel prennent forme les événements sonores : « Nous ne concevons pas la communication sonore comme quelque chose qui existerait indépendamment de l'homme et du contexte concret, comme un système abstrait» (J. Kawada, p. 1). "Le concept de culture sonore tel qu'il est étudié ici peut être défini comme suit : ensemble des communications sonores, vocales et/ou instrumentales, pratiquées dans différentes sphères de la vie d'une société » (idem, p. 5).

3 Une objection vient immédiatement à l'esprit concernant la place accordée à la langue dont la parole est l'expression sonore. Une définition si large de la "culture sonore " semble ne pas pouvoir prendre en compte la différenciation entre langue et expression sonore. Kawada considère que, en absence de l'écriture, toute communication sonore, 
verbale aussi bien que musicale, revient à la tradition orale. En effet ces textes soulignent les rapports étroits entre la sonorité de la parole parlée et la signification symbolique des sons instrumentaux alors même qu'ils concernent des sujets et des populations différents. Ainsi, sont notamment concernées la transmission de messages par le langage tambouriné, ou sifflé, et la relation directe entre les sons instrumentaux et les mouvements rythmés du corps qu'ils suscitent.

Bien évidemment, tout cela implique également la prise en compte des différentes fonctions que les événements sonores remplissent dans une société, ce qui entraîne, entre autre, la relativisation de la notion de beau en tant que critère d'évaluation artistique. Ayant étudié depuis vingt ans le langage tambouriné des Mosi de Tenkodogo au Burkina Faso et des Yoruba d'Oshogbo au Nigeria, Kawada considère qu'il est - plus qu'un succédané de la langue verbale - l'expression de l'ensemble de la configuration sonore d'une phrase qui s'exprime à travers une technique du corps culturellement conditionnée. En tant que système de communication sonore, le langage tambouriné est en même temps une extra-langue car il dépasse la langue verbale, et une intralangue car il est une langue à l'intérieur d'une langue verbale. Il faut donc prendre en compte les éléments de base de la communication pour étudier l'aspect fonctionnel du message sonore : l'émetteur, le récepteur, et le lieu de la transmission : «Pour analyser les fonctions sociales de la communication sonore à l'aide de cet appareil conceptuel, il faudrait y ajouter la distinction entre le récepteur réel du message et son destinataire virtuel. Cette dualité du récepteur (réel/virtuel) est très importante, me semble-t-il, pour comprendre la réalité de la culture sonore ouest-africaine » (J. Kawada, p. 12).

5 Pour expliquer ce point de vue, il prend l'exemple du nom de la personne qui peut être déclamé par autrui à haute voix, mais aussi par le tambour, pour que quelqu'un d'autre l'entende. L'auteur emprunte à la culture japonaise l'idée de " réunion en petit comité » ( $z a$ en japonais), c'est-à-dire un groupe limité de personnes qui se connaissent bien, et il emploie le néologisme de «syllogue » qui entraîne entre autre, la relativisation de la notion de beau en tant que critère différents narrateurs.

6 Après avoir esquissé la distribution des instruments en Afrique occidentale, l'auteur présente son hypothèse sur l'existence de deux complexes de culture sonore. Dans ce dessein, il part de la considération que les cultures sonores ne correspondent ni aux aires linguistiques ou politiques, ni aux groupes ethniques, mais qu'il est, par contre, possible de localiser des centres d'influence et des périphéries. Il propose ainsi des modèles d'analyse, qu'il appelle "complexes", regroupant un certain nombre d'éléments significatifs de la culture sonore ouest-africaine : "Les "complexes" ainsi conçus sont des modèles d'analyse élaborés par le chercheur à partir des matériaux pris dans les sociétés réelles étudiées, mais qui ne correspondent pas nécessairement toujours à la réalité. [...] Ce sont plutôt des outils heuristiques [...] pour découvrir les facteurs qui serviront à éclaircir les problèmes de la culture sonore non seulement pour l'Afrique occidentale, mais aussi pour l'humanité toute entière, en les comparant avec les modèles d'autres régions » (J. Kawada, p. 34).

7 Dans «Les Stambaâlî: leurs rites et leur culture sonore », Takaki Keiko analyse des cultes du Maghreb qui présentent des traits similaires à ceux de possession en Afrique subsaharienne. Créés à l'origine par des esclaves noirs, ces groupes rituels ont développé, sous l'influence de l'islam, des caractères syncrétiques : "L'appellation de ces groupes diffère d'une région à l'autre, mais ils partagent d'importants points de 
similitude : la croyance aux génies, une musique fortement rythmée, des danses en état de transe » (K. Takaki, p. 51).

Pour examiner l'organisation des membres de ces cultes, l'auteur prend notamment en considération le Stambaâlî, un groupe rituel noir de Tunisie, constitué par :

- un orchestre formé uniquement par des hommes,

- l'Arîfa, « celui qui sait », un personnage chamanique, souvent hermaphrodite et en marge de la société,

- le Bû Sa 'dîya, une sorte de bouffon qui représente un légendaire marabout noir.

Les rythmes musicaux sont liés aux esprits (appelés aujourd'hui jnûn) qui prennent possession du fidèle, provoquant l'état de transe. Dans le système de croyances du Stambaâlî, le culte des saints, considérés par les fidèles comme leurs ancêtres, comporte le sacrifice des boucs ou des taureaux noirs. Accompagnés d'une musique fortement rythmée et de danses en état de transe, ces rituels font fortement appel au corps et aux sensations physiques. C'est d'ailleurs dans les mouvements corporels de danse et de transe que l'on retrouve clairement les traits propres aux pratiques des groupes rituels noirs subsahariens, et ce bien que les membres du Stambaâlî soient à présent tous des musulmans. "Il est par conséquent très significatif d'observer que dans la culture populaire, la culture sonore, qui fait appel à l'inconscient (elle échappe pour cette raison à l'explication logique et théorique), a pu conserver plus facilement son héritage historique» (K. Takaki, p. 83). Takaki souhaite que cette étude sur les aspects syncrétiques des rituels religieux de l'Afrique subsaharienne puisse s'étendre de manière comparative à l'Amérique latine où le christianisme tient la place occupée au Maghreb par la culture arabo-islamique.

10 Nasaki Horiuchi analyse la culture sonore des Berbères originaires de la partie sudouest du Maroc («Between segmentation and de-segmentation: Sound expressions among the Berbers in the South region (Southwestern Morocco) »). La notion de culture sonore est pour lui un point de vue unifiant l'analyse de traits culturels disparates, normalement conçus en catégories opposées, tels que rural/urbain, tradition/ modernité, etc. C'est l'univers de vie des gens qui produisent et utilisent les sons qui est ainsi au centre de l'analyse, avec leurs propres expériences et feelings. Horiuchi appelle segmental les sons qui véhiculent, par des paroles signifiantes, des messages plus concrets, par rapport aux sons qu'il nomme de-segmental. Considérer les formules sonores du point de vue de l'équilibre trouvé entre segmentation et « désegmentation » facilite la prise en compte des interrelations entre expressions sonores jusque-là perçues comme des phénomènes différents: danse, littérature, récitation rituelle, musique populaire, etc. Ainsi l'auteur postule la nécessité de l'existence, dans chaque contexte social, d'un principe organisateur permettant la coexistence de sons par ailleurs indépendants.

11 Dans la suite du texte de Horiuchi, l'application de ces concepts à la description des différentes expressions sonores berbères souffre d'une certaine ambiguïté que l'auteur toutefois revendique comme intrinsèque à son objet d'étude. Les chants, les jeux instrumentaux, les déclamations, sont analysés dans des contextes différents, comme les cérémonies de mariage, la tradition des ménestrels, les modernes bandes musicales de rais ou la récitation du Coran. En conclusion, Horiuchi précise que son but n'était pas celui de décrire la culture par une opposition binaire arbitraire entre segmentation et désegmentation, car de toute façon aucun événement culturel ne rentre parfaitement dans l'une de ces catégories. Son intention était plutôt, d'une part, de 
mettre en évidence comment différents aspects peuvent coexister dans la même personne ou dans la même société, et, d'autre part, de promouvoir la fonction heuristique de son schéma, qu'il estime être à même de générer une nouvelle compréhension.

Avec une approche ethnolinguistique, le texte de Oger Kabore (« Les chansons d'enfant Moose : signification socioculturelle d'un mode d'expression des jeunes filles en milieu rural ») aborde un thème peu étudié, les chansons d'enfants, dont la compréhension profonde demande un fort degré d'intégration dans le contexte où ces chants prennent vie et se transforment. Cela valide la démarche de Kabore qui, depuis une dizaine d'années, mène dans son propre pays, le Burkina Faso, des recherches sur la littérature orale. Il nous propose ici l'analyse des chansons des jeunes filles de la province de Kouritenga: «Si nous voulons focaliser notre attention sur les chansons des jeunes filles, c'est en raison du fait qu'elles recèlent des trésors inestimables pouvant nous aider à comprendre l'organisation sociale et en particulier, l'image de la femme dans cette société » (O. Kabore, p. 123).

13 À travers l'analyse des chansons enfantines, l'auteur touche aussi au système pédagogique de transmission des savoirs et valeurs d'une société où la parole, et à plus forte raison la parole chantée, est chargée des forces ambivalentes qui la rattachent à une dimension métaphysique sacrée. Ces chansons de jeunes filles sont en même temps des chansons populaires et de danse, et l'analyse de leur contexte d'exécution donnera la clé d'interprétation des différentes fonctions qu'elles remplissent. Se questionnant sur ce qui séduit les enfants dans ces chansons, Kabore trouve deux explications possibles. L'une tient aux qualités littéraires des textes chantés qui en font des véritables poèmes oraux, l'autre à la stratégie du système éducatif traditionnel qui prépare ainsi les jeunes à une intégration équilibrée dans la société : «Le grand défi lancé aux pédagogues africains consiste à œuvrer pour une intégration judicieuse de ces riches acquis de la culture sonore africaine dans la pédagogie moderne actuelle » (id., p. 140).

C'est dans le patrimoine musical nigérien que puise l'article de Mahaman Garba sur les griots du Niger ("Fonctions sociales des griots hawsa du Niger ») - aire musicale que Gilbert Rouget appelle "soudanaise ou négro-islamique ». Bien qu'appartenant à une société sédentaire, les griots, ainsi appelés en Occident, sont une caste d'artistes musiciens itinérants. De ce fait, ils contribuent aux échanges musicaux dans une région pluriethnique où la différence est traditionnellement marquée entre musique des populations sédentaires et nomades : "Il est intéressant de constater, qu'à chaque groupe ethnique correspond une culture musicale particulière et que la manière des joueurs d'instruments s'en ressent. C'est ce qui nous fait dire qu'il faudrait plutôt parler des musiques traditionnelles au Niger et non de la musique traditionnelle » (M. Garba, p. 160).

En s'interrogeant sur les phénomènes actuels de déculturation et d'acculturation, l'auteur constate quatre typologie d'attitudes à l'égard de la musique. L'une alimente son mépris par les préjugés traditionnels sur le statut inférieur du musicien. Elle freine ainsi le talent des jeunes et par là même tout développement de la musique traditionnelle, les fils des griots cachant de plus en plus leurs origines et reniant leur caste. L'islam, pour sa part, condamne la musique comme activité mondaine. Des attitudes différentes distinguent les classes d'âge: les personnes âgées du côté de la musique traditionnelle, les jeunes du côté de la musique occidentale, afro-américaine 
ou world music, qu'ils appellent musique de variété, mais que Garba suggère, suivant Aubert, de qualifier de musique populaire moderne. Vient enfin l'attitude de ségrégation entre musiciens traditionnels de ville et de village, et entre musique sédentaire et nomade. Par la suite, différentes typologies des musiciens ou des déclamateurs généalogistes sont dressées dans le contexte socioculturel du peuple hawsa. Ces classifications sont à l'image de cet article, qui semble parfois imprécis et laisse un peu sur sa faim, mais où on trouve aussi beaucoup d'informations ponctuelles. La richesse du sujet traité, il est vrai, se prête mal à une catégorisation rigoureuse. Ichiro Majima, dans son article "Voix de masques sans visage : "Maania" chez les Dan du Danané-Sud (Côte-d'Ivoire) » introduit l'intéressante notion de "masque sonore ». Si l'aire communément appelée Central West Atlantic Region (CWA) présente plusieurs ethnies et cinq groupes linguistiques, elle se caractérise tout de même par la diffusion d'activités rituelles des masques liées aux sociétés de pouvoir de type Poro/Sande. Le masque représentant toujours un esprit, Majima distingue deux catégories : corporelle et sonore (soit visuelle et auditive). Si l'on peut regrouper ces diverses matérialisations visuelles des génies dans la catégorie des "masques corporels", la représentation spirituelle du "génie audible mais invisible ", autrement dit le "masque sonore », est beaucoup plus répandue et rituellement plus révérée en CWA (I. Majima, p. 240). La mise en scène du masque sonore est toujours basée sur l'interdiction faite aux non initiés de voir le masque : ils en entendent seulement la voix. La voix du génie peut ainsi être produite par des hommes sans masque facial mais toujours cachés.

En concluant sur la prédominance de l'aspect « vocal » du masque dans l'ensemble de la CWA, l'auteur s'interroge: "Voir dans ce phénomène une version africaine du mot latin persona dérivé de per sona, serait-il considéré comme un manquement à la démarche orthodoxe de l'ethnographie...?». Intriguée par ce questionnement rhétorique, j'ai essayé d'approfondir la question car la déduction me semblait plutôt hasardeuse. Cependant, sona est en italien la troisième personne du verbe sonare (ou suonare), jouer un instrument, produire du son. Toutefois, l'étymologie de persona, pour le Devoto-Oli (dictionnaire de la langue italienne) tout comme pour Le Robert, montre la dérivation de l'étrusque phersum, masque de théâtre, mot devenu ensuite individu. Je connaissais l'étymologie du latin imago, qui désigne le masque funéraire, mais j'étais surprise de retrouver le masque à l'origine d'individu, bien que l'identification d'une racine concernant le «son » demeure arbitraire, me semble-t-il.

Sur le plan linguistique encore, Majima souligne le paradoxe par lequel le terme générique de "masque » n'existe pas chez les Dan - dont ce texte examine le masque sonore appelé «Maania » - alors que cette population est fort connue pour la grande valeur artistique de leurs masques faciaux. Différents mots désignent le corps de l'homme déguisé, la «tête de génie » (masque facial), ou le " génie nocturne » (masque sonore), mais surtout il existe plusieurs sortes de masques, corporels aussi bien que sonores, que l'auteur décrit et classifie dans plusieurs tableaux concernant la morphologie des masques ainsi que les instruments qui leur correspondent, leurs fonctions sociales et l'organisation dans le système lignager. L'analyse des relations entre société de pouvoir et masques chez les Dan montre une structure fortement hiérarchisée.

19 Le savoir et les techniques concernant la représentation des "génies" sont soigneusement gardés par les hommes circoncis chez les Dan. Il s'agit des « secrets des hommes » [...], étroitement liés à la société masculine de pouvoir Go (I. Majima, p. 247). 
20 Maania est le grand masque sonore qui ne sort qu'à des années d'intervalle par une nuit de la nouvelle lune. En cette occasion, tous les villageois - sauf les membres masculins du lignage chargé de Maania - doivent rester enfermés chez eux, silencieux. L'analyse de Majima s'enrichit de comparaisons avec des ethnies proches, notamment pour ce qui est des recherches historiques concernant Maania, et demeure très suggestif pour l'analyse des représentations en général. Les masques corporels et sonores, souvent localement différenciés par l'opposition binaire "jour/nuit » ou "visible/invisible », sont dans l'analyse de l'auteur en dernière instance un système de contrôle des informations. S'appuyant aussi sur nombre d'études d'ethnomusicologues, comme Zemp ou Bellman, Majima constate donc que le contrôle de l'information passe par trois canaux et leurs mises en scène correspondantes: visuelle (masque corporel et facial), auditive (masque sonore) et verbale : « La mise en scène du masque est donc une forme de contrôle de l'information qui en voile une partie et dévoile le reste en même temps » (id., p. 291).

21 "Quand le reggae se marie avec la culture de la rue... », de Hiroyuki Suzuki, analyse un morceau de reggae africain interprété par Tangara Speed Ghôda, un chanteur d'Abidjan considéré comme le porte-parole des jeunes qui cherchent leur pain quotidien dans la rue. Lui-même issu de ce milieu, son morceau «Loubard Feeling» reflète, comme l'ensemble du reggae abidjanais, l'identité de ces jeunes qui ont aussi développé un mode de communication propre, fondé sur une langue véhiculaire et des gestes souvent inspirés par les films de karaté. Après un historique du reggae abidjanais et du rôle des médias locaux dans sa diffusion, l'auteur donne un aperçu de la culture de ces jeunes hommes - de dix à trente ans - qui trouvent leurs moyens de subsistance dans le secteur informel du travail ou dans le crime. La "rue» est une microsociété multiethnique où s'établissent des relations sociales différentes de celles du milieu traditionnel ou de la société moderne, ce qui justifie la nécessité d'un langage commun, le nouchi. Chantés en plusieurs langues africaines, en français et en nouchi, les textes des chansons du reggae abidjanais intègrent une critique sociopolitique en même temps qu'ils reflètent des traits caractérisant ce milieu (crime, violence, drogue, prostitution).

«"Loubard" est un mot français signifiant "jeune voyou" "jeune délinquant" [...]. Mais dans la rue d'Abidjan [...] est un jeune qui met l'accent sur son apparence physique [...] fait montre d'un corps bien musclé [...] cherche souvent la bagarre parce que c'est la meilleure occasion pour lui de prouver sa force [...]. Tangara Speed Ghôda. Devenu chanteur de reggae, il adopte le style "rasta", mais il continue à s'identifier au "loubard" » (H. Suzuki, p. 318).

22 À l'instar de la plupart des chanteurs de reggae abidjanais, Tangara Speed Ghôda est en même temps l'auteur et le compositeur de ses chansons. Après des informations sur sa vie, Suzuki décrit le style de composition de cet auteur, en détaillant le processus de création de la chanson « Loubard Feeling ».

23 À la fin de cet ouvrage, le texte de Kenichi Tsukada, «Drumming, Onomatopoeia and Sound Symbolism Among the Luvale of Zambia ", juge inadéquats les termes jusqu'à maintenant utilisés pour le domaine de la transmission orale, aussi bien que cette notion même : «In recent years, there has been an important development in the study of "sound cultures" with particular reference to the "oral transmission" of instrumental music. This new turn has been based on an increasing concern with the issue of sound symbolism. [...] The system of "oral transmission" has been explored from time to time with such designations as "nonsense syllables", "oral mnemonic", 
"oral notation", and even "solfege". [...] Even the notion of "oral transmission" may be problematic, because the practice is not always concerned with transmision as such. A more neutral, more universally applicable terme is required, and I propose the terme "verbal representation of instrumental sound" (K. Tsukada, p. 349).

Mise à part toute autre considération, on pourrait s'étonner que pour remplacer deux mots on doive utiliser toute une phrase, qu'en effet l'auteur doit ensuite abréger en VRIS et puis en VR, abréviation de l'abréviation. Au début, Tsukada s'intéresse au VR par rapport à l'étude des tambours parlants, chez les Luvale de la Zambie. C'est à partir de ses propres erreurs d'exécution qu'il commence à soupçonner que le tambouriner luvale construit des syllabes basées sur la qualité du son du tambour : « I expected that such aspects could be uncovered by analyzing the syllables in terms of sound symbolism » (id., p. 351).

L'auteur mène donc une enquête sur questionnaire et recueille plusieurs idéophones, en analysant notamment les onomatopées, pour investiguer le symbolisme sonore luvale. Population matrilinéaire et virilocale de cultivateurs, les Luvale ont développé une riche tradition des tambours rituels, et trois techniques de percussion qui associent à chaque son un timbre et une hauteur spécifiques. De son enquête - dont il présente ici des exemples méthodologiques, des tables analytiques, des figures et des spectrogrammes -, Tsukada conclue que dans le tambouriner luvale il y a systématiquement symbolisme sonore mais que la relation entre le système de VR et le symbolisme sonore n'est pas une relation directe mais inverse : " "Hence" the inversion phenomenon in sound symbolism » (id., p. 385).

Une autre donnée intéressante dans la recherche de Tsukada est le constat que de petites différences sonores, mises en évidence par l'analyse spectrographique mais que l'auteur n'arrivait pas à apprécier, étaient parfaitement identifiées par les Luvale: "The Luvale, on the other hand, responded to these differences with surprising precision by consistently using various VR syllables » (id., p. 385).

Des questions restent donc ouvertes concernant la signification du phénomène de l'inversion dans le symbolisme sonore aussi bien que les caractéristiques culturelles des perceptions des sons car l'auteur, pour expliquer certaines données problématiques de l'enquête, arrive à se demander s'il ne faudrait pas conclure pour les Luvale à une perception « super-sensorielle » qui agirait au niveau inconscient.

Les concepts, proposés dans l'ensemble de ces textes, de "culture sonore » et de "communication sonore" semblent efficaces pour faciliter le processus d'émancipation de l'ethnomusicologie contemporaine par rapport à une idée encore trop ethnocentrique de «musique». Ainsi, l'ethnomusicologie continue d'acquérir de plus en plus d'autonomie par rapport à sa matrice musicologique et cimente sa quête de spécificité en approchant davantage, par ses méthodes aussi bien que par ses objets d'étude, le domaine proprement anthropologique. 